\title{
Análisis de la implementación del Programa Nacional Integral de Sustitución de Cultivos Ilícitos (PNIS): 2017-2019*
}

\author{
Analysis of the implementation of the National \\ Comprehensive Program for the Substitution of Illicit \\ Crops (PNIS): 2017-2019
}

\section{Valeria Mejía Hidalgo**}

Artículo de investigación

Fecha de Recepción: 14 de enero de 2021

Fecha de Aceptación: 28 de febrero de 2021

\section{Para citar este artículo:}

Mejía Hidalgo, V. (2021). Análisis de la implementación del Programa Nacional Integral de Sustitución de Cultivos llícitos (PNIS): 2017-2019. Revista Análisis Jurídico-Político, 3(5), 171-199. https://doi.org/10.22490/26655489.4530

\section{Resumen}

El Programa Nacional Integral de Sustitución de Cultivos Ilícitos (PNIS) se deriva del Acuerdo de Paz entre el Gobierno de Colombia y la guerrilla FARC-EP. En sus dos primeros años ha tenido un desempeño suficiente en la reducción de hectáreas de hoja de coca cultivadas en el país, pero insuficiente en la provisión de las respuestas para el mejoramiento sostenido de la calidad de vida

\footnotetext{
* El presente artículo es producto de la tesis de grado del Máster Universitario en Relaciones Internacionales, Seguridad y Desarrollo de la Universitat Autònoma de Barcelona. Su título original es Análisis del Programa Nacional Integral de Sustitución de Cultivos llícitos (PNIS) de Colombia en el marco de la implementación del Acuerdo de Paz: 2017-2019.

** Politóloga por la Universidad Nacional de Colombia, magíster en Relaciones Internacionales, Seguridad y Desarrollo por la Universitat Autònoma de Barcelona. Correo electrónico: valeria.mejiah@e-campus.uab.cat; vmejiah@una.edu.co oRCID: https://orcid.org/0000-00028132-0588
} 
de la población cocalera. Los desfases en la ejecución de sus componentes, la inestabilidad de las condiciones de seguridad y las falencias de articulación con las estrategias de la Reforma Rural Integral (RRI) han impactado el aporte del PNIS a la transformación del campo colombiano como lo contempla el Acuerdo de Paz.

Palabras clave: Acuerdo de Paz, Colombia, cultivos ilícitos, desarrollo rural, hoja de coca, implementación, PNIS

\section{Abstract}

The National Comprehensive Program for the Substitution of Illicit Crops (PNIS) is part of the Peace Agreement signed by the Colombian government and the Revolutionary Armed Forces of Colombia-People's Army (FARC-EP guerrillas). During its two first years, the program has contributed to diminishing coca bush cultivation, but it hasn't provided enough responses to improve the quality of life for the communities involved in the substitution process. Gaps in the implementation of its components, instability of security conditions, and lack of articulation with the rural development strategies (RRI) have impacted the PNIS contribution to the transformation of the Colombian countryside.

Key words: coca bush cultivation, Colombia, illicit crops, peace agreement implementation, PNIS, rural development

\section{INTRODUCCIÓN}

La producción de cultivos de hoja de coca para fines ilícitos es una problemática que el Estado colombiano ha afrontado desde la década de los ochenta. Hasta la fecha de este documento ninguna estrategia antidrogas ha funcionado, pues Colombia ha sido el primer productor mundial de hoja de coca desde los noventa, casi ininterrumpidamente. Según las últimas estimaciones globales de la Oficina de las Naciones Unidas contra la Droga y el Delito 
(UNODC) (Sistema integrado de monitoreo de cultivos ilícitos [SIMCI], 2020), en el año 2017 el país concentró el $70 \%$ del área sembrada con cultivos de coca y contó con el $50 \%$ de los hogares cultivadores de esta planta en el mundo.

Aun así, desde enero de 2017 se adelanta una nueva estrategia en el territorio colombiano que apunta a desincentivar la producción de hoja de coca mediante la provisión de alternativas económicas para las familias involucradas en el cultivo y recolección: el Programa Nacional Integral de Sustitución de Cultivos Ilícitos (PNIS). Su origen se enmarca en punto 4 del Acuerdo de Paz ${ }^{1}$ suscrito entre el Gobierno colombiano y las Fuerzas Armadas Revolucionarias de Colombia-Ejército del Pueblo (FARC-EP) a finales del año 2016, en el que por primera vez en la historia de los procesos de paz del país ${ }^{2}$ se abordó el fenómeno de los cultivos ilícitos como uno de los ejes para la construcción de paz.

La lógica del programa se resume así: el Gobierno se compromete a poner en marcha todas las medidas para el proceso de sustitución, mientras las comunidades asumen la responsabilidad de erradicar sus plantaciones y no volver a incurrir en ninguna actividad relacionada con los cultivos ilícitos (Presidencia de la República, 2017). En este sentido, la cuota del PNIS a la transformación del campo colombiano radica en su función como estrategia de transición que soporte tanto el cambio de las fuentes de sustento económico de las familias cultivadoras y no cultivadoras, como la reconversión laboral de la población recolectora, lo que mitigaría el riesgo de resiembra y de su revinculación con la producción de cultivos ilícitos.

\footnotetext{
1 El Acuerdo se conforma por seis puntos: 1) Reforma Rural Integral; 2) participación política; 3) cese al fuego y de hostilidades bilateral y definitivo, y la dejación de armas; 4) solución al problema de las drogas ilícitas; 5) víctimas; y 6) mecanismos de implementación y verificación.

2 Según la Fundación Ideas para la Paz (2014), la problemática de la producción de cultivos para fines ilícitos hizo parte de los intentos de negociación entre las FARC-EP y el Gobierno colombiano durante las presidencias de Belisario Betancur (1982-1986) y Andrés Pastrana (1998-2002), pero solo en esta oportunidad se concibió como un punto específico en la agenda.
} 
El universo poblacional del PNIS es de 99097 familias ubicadas en 56 municipios de 14 departamentos $^{3}$ de Colombia, cuya atención entre enero de 2017 y 31 de diciembre de 2019 ha representado la inversión de 1111887519357 cop —más de 320000000 USD(Consejería Presidencial para la Estabilización y Consolidación, 2020). Más allá de las cifras, la realidad muestra que la implementación del programa tiene niveles de avance diferenciados en cada territorio debido a la existencia de problemas de fondo, como la escasa presencia sostenida del Estado, la desconexión con la Reforma Rural Integral (RRI) y la inestabilidad en materia de seguridad y protección de las comunidades (Bernal, Garzón y Gélvez, 2019).

Este artículo tiene como objetivo analizar el aporte del PNIS a la construcción de paz en Colombia, a partir de los adelantos en la ejecución de sus componentes. Para ello, se plantean dos propósitos específicos: 1) describir los principales avances y dificultades de la implementación del esquema de sustitución durante el período 2017-2019, haciendo énfasis en lo pactado en el literal 4.1.3 del Acuerdo de Paz; y 2) identificar los principales factores que configuran desafíos para el resto de la vigencia del Programa, teniendo en cuenta la experiencia acumulada a lo largo de la temporalidad de estudio.

La pregunta central que se quiere responder es: ¿en qué sentido lo implementado del PNIS entre 2017 y 2019 ha contribuido o no al proceso de construcción de paz en Colombia, en especial con respecto al fomento del desarrollo rural? Adicionalmente, se consideran dos cuestionamientos complementarios: ¿cuál ha sido el desempeño del PNIS en sus dos años de ejecución? y ¿cuáles han sido sus avances y dificultades?

A modo de hipótesis se plantea que el PNIs ha sido la estrategia más ambiciosa de sustitución implementada en Colombia para reducir los cultivos ilícitos. En sus dos años de ejecución — de los

3 Los municipios pNIS están en los departamentos de: Antioquia, Arauca, Bolívar, Caquetá, Cauca, Córdoba, Guainía, Guaviare, Meta, Nariño, Norte de Santander, Putumayo, Valle del Cauca y Vichada. 
diez proyectados para su vigencia- ha logrado atender casi a 100000 familias ubicadas en municipios que concentran el $65 \%$ de los cultivos de hoja de coca ${ }^{4}$ del país (Oficina de las Naciones Unidas contra la Droga y el Delito [UNODC], 2020). Sin embargo, en el proceso han surgido dificultades de cara a la consolidación del Programa a mediano y largo plazo — desfases en la ejecución de los diferentes componentes del esquema de sustitución, inestabilidad de las condiciones de seguridad territoriales y falencias de articulación con las estrategias de la RRI-, que podrían afectar su aporte a la transformación del campo colombiano como lo contempla el Acuerdo de Paz.

El documento se estructura en dos apartados: 1) pauta de análisis, donde se expone la metodología diseñada y se describen los elementos principales del PNIS; y 2) seguimiento a la implementación de los componentes del PNIs en el período 2017-2019. El lector encontrará, a modo de cierre, las conclusiones que resaltan las ideas fuerza distribuidas a lo largo del texto.

\section{Pauta de ANÁlisis}

El seguimiento a la ejecución de los componentes del PNIS contemplados en el subpunto 4.1. del Acuerdo de Paz y en su Plan marco de implementación ${ }^{5}$ (PMI) es el criterio general que rige el presente análisis. Entiéndase por componentes a los elementos que integran el Programa, los cuales se definen así:

- Acuerdos con las comunidades: proceso de formalización de los compromisos entre el Gobierno nacional y la población involucrada en el cultivo y recolección de plantaciones ilícitas. El Ejecutivo se responsabiliza de la ejecución del Plan de atención

4 El Sistema integrado de monitoreo de cultivos ilícitos (SIMCI), manejado por UNODC, es el encargado de realizar anualmente el censo de áreas afectadas por los cultivos ilícitos en Colombia. El informe se presenta en el segundo semestre de cada año y contiene las cifras del año inmediatamente anterior.

5 Es el documento de planificación que "concretó los compromisos de política necesarios para lograr" lo pactado en el Acuerdo de Paz (Consejo Nacional de Política Económica y Social, 2018). 
inmediata (PAI) y la construcción e implementación de los Planes integrales de sustitución y desarrollo alternativo (PISDA). Mientras que las comunidades asumen el compromiso de no incurrir en la resiembra, ni en cualquier otra actividad relacionada con los cultivos ilícitos.

- Plan de atención inmediata: grupo de medidas de apoyo inmediato para facilitar el tránsito de las personas cultivadoras, no cultivadoras y recolectoras hacia economías legales que garanticen su sustento y seguridad alimentaria.

- Planes integrales de sustitución y desarrollo alternativo: documentos de planeación que identifican las necesidades, oportunidades y prioridades para el desarrollo de los municipios focalizados por el PNIS. Se construyen mediante un proceso de planeación participativa con enfoque de abajo hacia arriba (bottom-up), en el que intervienen las comunidades, el Gobierno nacional y las autoridades locales.

- Tratamiento penal diferencial: trámite de ajustes normativos para reducir la judicialización y el encarcelamiento por los delitos de conservación o financiación de cultivos ilícitos, según lo establece el Código Penal colombiano vigente. Su población objeto son los pequeños cultivadores de plantaciones ilícitas que manifiestan su decisión de renunciar a la producción de dichos cultivos.

- Condiciones de seguridad para la sustitución: medidas de protección para garantizar la vida y el bienestar de las comunidades afectadas por los cultivos ilícitos frente a cualquier coacción o amenaza presentada en el marco del proceso de sustitución.

- Sustitución en parques nacionales naturales: conjunto de estrategias diferenciales para la erradicación voluntaria de cultivos ilícitos en las áreas de protección ambiental especial, con miras a garantizar su restauración, protección y el sustento de las familias beneficiarias del Programa.

Conviene mencionar que, para el texto, la implementación del Programa inicia con la construcción de acuerdos colectivos de sustitución entre el Gobierno y las comunidades, pues de ahí se desprende la vinculación individual posterior de las familias al 
proceso de sustitución, la erradicación voluntaria de las plantaciones y, por lo tanto, su acceso a los demás componentes descritos.

La temporalidad de la investigación se enmarca en los dos mandatos presidenciales que han asumido la implementación del PNIS desde su creación en enero de 2017 hasta el 31 de diciembre de 2019. En este sentido, al mencionar las acciones ejecutadas entre el 27 de enero de 2017 y el 6 de agosto de 2018 se hace referencia a lo adelantado por la administración de Juan Manuel Santos. Mientras que cuando se abordan los hallazgos comprendidos entre el 7 de agosto de 2018 y el 31 de diciembre de 2019 se hace alusión al período presidencial de Iván Duque.

La información fue recolectada por medio del análisis bibliográfico de dos tipos de fuentes: 1) los informes oficiales y bases de datos de las instituciones encargadas de la implementación y seguimiento del PNIS y del Acuerdo en general; y 2) los informes públicos o balances realizados por think tanks y organizaciones internacionales en dicha materia.

Por último, se aclara que este estudio no debe considerarse como una evaluación completa de los resultados del PNIS, debido a que los dos años de ejecución analizados no proveen información suficiente para medir su impacto. Sumado a ello, dicha tarea corresponde a las instituciones estatales encargadas de su implementación y a las instancias que contempla el punto 6 del Acuerdo de Paz, como la Comisión de seguimiento, impulso y verificación a la implementación del Acuerdo Final, el Instituto Kroc de Estudios Internacionales de Paz de la Universidad de Notre Dame y, especialmente, el punto 4 para la UNODC.

\subsection{Descripción del Programa Nacional Integral de Sustitución de Cultivos llícitos}

El objeto formal del PNIS es:

[...] promover la sustitución voluntaria de cultivos de uso ilícito, a través del desarrollo de programas y proyectos para contribuir a la superación de condiciones de pobreza y marginalidad 
de las familias campesinas que derivan su subsistencia de los cultivos de uso ilícito. (Presidencia de la República, 2017)

El Decreto 896 de 2017 contempla que la entidad encargada de su implementación es la Dirección de Sustitución de Cultivos Ilícitos, adscrita al Departamento Administrativo de la Presidencia (Dapre), e instituye una serie de instancias nacionales y territoriales para la ejecución, coordinación y gestión del Programa, que garanticen el principio de construcción conjunta, participativa y concertada que contempla el Acuerdo de Paz para este punto (Presidencia de la República, 2017). Adicionalmente, tanto el acuerdo como el decreto mencionado definen que la oferta del PNIS se enfoca en las personas que se encuentran en las actividades primarias de la producción de narcóticos; es decir, en las labores de cultivo y cosecha de la hoja de coca.

Como se ilustra en la figura 1, la ruta de intervención concertada inicialmente entre el Gobierno Santos y los voceros de la extinta guerrilla de las FARC-EP se proyectó a 24 meses, que comprenderían los siguientes pasos: 1) identificación de zonas de intervención; 2) socialización del PNIS y suscripción de acuerdos colectivos de sustitución; 3) vinculación individual de las familias; 4) desembolso del primer pago de asistencia alimentaria inmediata ${ }^{6}$; 5) verificación censal por la UNODC ; 6) segundo desembolso de seguridad alimentaria inmediata; 7) inicio del proyecto de seguridad alimentaria y asistencia técnica; 8) inicio de los proyectos productivos (Consejería Presidencial para la Estabilización y la Consolidación, 2019). Se contempló una atención de 12 meses para las familias recolectoras mediante su vinculación a programas de empleo rural temporal.

6 Son seis desembolsos bimensuales, por un monto de 2000000 cop cada uno.

7 La unODC tiene la función de verificar y monitorear el cumplimiento de compromisos de las comunidades frente al pNIs. Para ello desarrolla cuatro misiones: 1) el diagnóstico territorial de las veredas y la constatación del número de hectáreas comprometidas por los cultivadores para sustitución; 2) la verificación del compromiso de erradicación voluntaria después del primer pago de asistencia alimentaria; 3) la comprobación del cumplimiento del compromiso de no resiembra y el avances de los otros componentes del pNIs después de un año del desembolso de la primera asignación de asistencia alimentaria; y 4) la verificación final una vez se entregan todos los recursos del PAl familiar (UNODC, 2020). 
Figura 1. Hoja de ruta inicial del PNIS

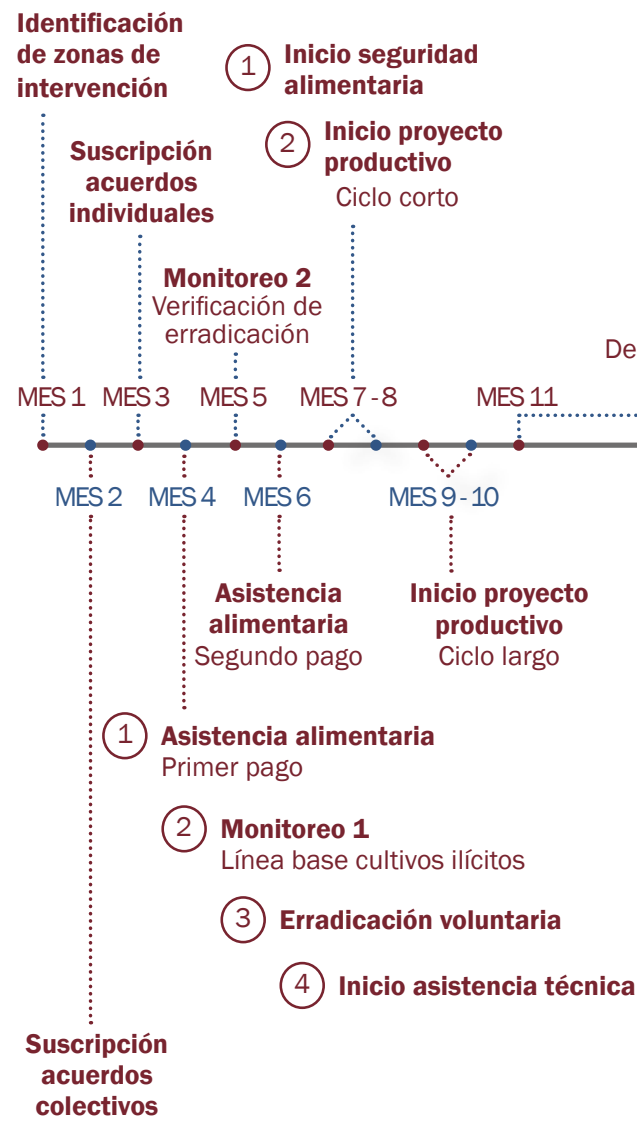

Fuente: elaboración propia, a partir del Informe ejecutivo consolidado $\mathrm{n}^{\circ} 13$ del pNIS.

Entre noviembre de 2018 y abril de 2019, el Gobierno Duque emprendió un proceso de reingeniería al funcionamiento del Programa. De acuerdo con los informes de gestión de la Consejería Presidencial para la Estabilización y Consolidación ${ }^{8}$ (2019)

8 Es la sucesora de la Alta Consejería para el Posconflicto y la responsable de articular interinstitucionalmente todos los aspectos relacionados con la implementación del Acuerdo de Paz (Presidencia de la República, 2019). 
(Gobierno de Colombia, 2020), una de las principales acciones desarrolladas ${ }^{9}$ fue la modificación de los tiempos de la ruta del PNIS. Se pasó de una intervención proyectada a 24 meses a una de 29 meses, que empiezan a correr desde el paso de la verificación censal por parte de UNODC y no desde la identificación de las zonas de intervención, como se muestra en la figura 2.

Figura 2. Hoja de ruta ajustada del PNIS

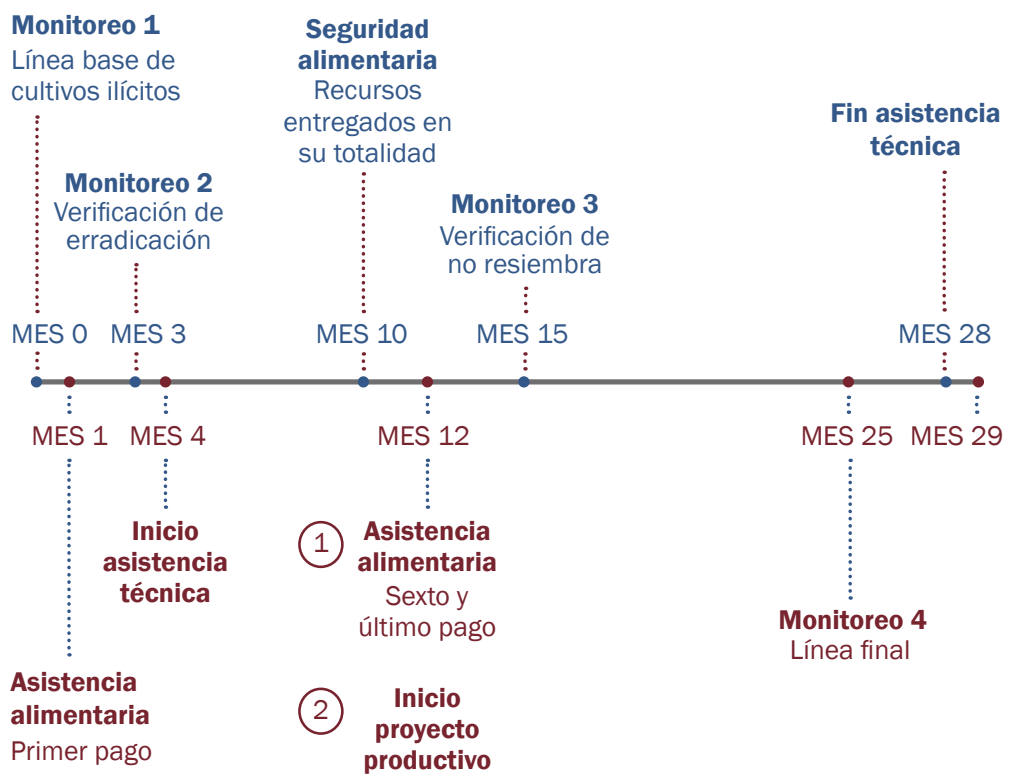

Fuente: elaboración propia a partir de los informes PNIS de la Consejería.

Por último, otra acción significativa para la operación del Programa ocurrió el 22 de noviembre de 2019 con la expedición del Decreto 2107 que estipula: el traslado de la Agencia de Renovación del Territorio (ART) del sector agricultura y desarrollo rural al

9 El resto de las acciones se resumen en: 1) estructuración de manuales operativos y procedimientos para cada componente del Programa; 2) mejora del sistema de información del PNIS; y 3) rediseño de la arquitectura institucional y fortalecimiento de los equipos territoriales (Gobierno de Colombia, 2020). 
Departamento Administrativo de la Presidencia (Dapre). También, el cambio de la Dirección de Sustitución de Cultivos Ilícitos de la Consejería a la ART con el objetivo de garantizar la articulación entre la RRI y el PNIS (Gobierno de Colombia, 2020).

\section{Implementación del Programa Nacional Integral de Sustitución de Cultivos Ilícitos}

En cada uno de los 56 municipios priorizados el PNIs ha alcanzado niveles de avance diferenciados. Ha afrontado problemas comunes y tiene desafíos por delante para cumplir con el compromiso que el Estado asumió con las familias beneficiarias y con su función " [...] como componente de la política de desarrollo rural enfocado en la transición" (Garzón y Suárez, 2018). A continuación, se describe el estado de implementación de cada uno de los componentes del Programa.

\subsection{Acuerdos con las COMUNIDADES}

La suscripción de acuerdos de sustitución entre el Gobierno y las comunidades se efectuó entre febrero de 2017 y abril de 2018. En dicho período, la Dirección - junto con el acompañamiento del partido de los Comunes ${ }^{10}$ — realizó aproximadamente 850 jornadas de socialización e información.

[...] en coordinación con los entes territoriales y organizaciones sociales que conforman las instancias nacionales del pNIs en 12 departamentos (Putumayo, Norte de Santander, Guaviare, Antioquia, Córdoba, Nariño, Caquetá, Meta, Vichada, Valle del Cauca, Cauca y Arauca) a fin de dar a conocer el alcance del PNIS y la adecuación del mismo al contexto territorial. (Departamento Administrativo de la Presidencia de la República, 2017)

10 Nombre adoptado en enero de 2021 por el partido político conformado por los exmiembros de las FARC-EP como parte de la implementación del Acuerdo de Paz. 
Según la Fundación Ideas para la Paz (FIP), en los departamentos de Cauca, Meta, Putumayo y Antioquia el acompañamiento de los exmiembros de las FARC-EP contribuyó a "generar confianza y facilitar que los campesinos [tomaran] la decisión de inscribirse en el PNIS" (Álvarez Vanegas y Garzón, 2017). No obstante, el mismo think tank ha reiterado en varias oportunidades (Álvarez Vanegas y Garzón, 2017; Garzón y Suárez, 2018; Garzón y Gélvez, 2018b) que el doble rol de la antigua guerrilla como parte del proceso de toma de decisiones estratégicas del Programa y, al mismo tiempo, como acompañante de la firma de acuerdos se tradujo en el surgimiento o la escalada de tensiones sociales en los territorios donde las organizaciones sociales reclamaban mayor independencia en la interlocución con el Gobierno nacional. Un ejemplo de ello es la subregión del Catatumbo — departamento de Norte de Santander-.

Producto de las socializaciones, según los informes de rendición de cuentas del Dapre (2017; 2018), desde enero de 2017 y el primer semestre de 2018, la Dirección logró la suscripción de 63 acuerdos colectivos de sustitución a nivel municipal, departamental o regional, que proyectaban la vinculación estimada de 135000 familias y 120000 hectáreas al proceso de sustitución de cultivos de hoja de coca. Sobre esta base, el ente administrativo procedió a la vinculación individual de las familias: en 2017 ingresaron al programa 24953 núcleos familiares en calidad de cultivadores, no cultivadores y recolectores; mientras que, hasta julio de 2018, la cifra ascendió a 77659 familias. La cobertura territorial del PNIS también incrementó de 19 municipios en el año 2017 a 51 municipios en el primer semestre del año siguiente.

Con el cambio de Gobierno nacional en agosto de 2018, la Consejería (2019) reportó que, tras la revisión de la documentación del PNIS, el universo real de acuerdos colectivos fue de 106 suscritos $^{11}$ entre febrero de 2017 y abril de 2018, lo que contemplaba como

11 De los cuales 71 tenían cobertura municipal, 16 suscritos en consejos comunitarios, 13 aglomeraban núcleos veredales, 4 constaban de cobertura regional, 1 abordaba el territorio de un resguardo indígena y 1 cubría área de Parques Nacionales Naturales (PNN). 
potenciales beneficiarias a 188036 familias en 98 municipios. Las primeras decisiones del Gobierno Duque fueron priorizar la atención de las familias que habían suscrito vinculaciones individuales con la administración Santos y cerrar la posibilidad de incluir a nuevas familias, así se encontraran proyectadas en los acuerdos colectivos. Tanto para estas familias, como para la población que no tuvo ninguna relación con el PNIS se crearían otros esquemas de sustitución voluntaria ${ }^{12}$ (Soto, 2020).

Lo que sí se consolidó fue el número definitivo de población y los territorios que el Programa atenderá durante su vigencia: la cobertura territorial se delimitó a 56 municipios de 14 departamentos, con un universo población de 99097 núcleos familiares, siendo 67237 cultivadores de hoja de coca, 16860 recolectores y 15000 no cultivadores que habitan en territorios afectados por el narcotráfico (UNODC, 2020).

Este componente también comprende la posibilidad de ejecutar las modalidades de erradicación forzada cuando la sustitución no sea posible. Los acuerdos colectivos del pNIS lo contemplaron en tres ocasiones:

i) cuando las familias se nieguen a inscribirse individualmente en el programa y manifiesten su intención de no sustituir voluntariamente los cultivos de uso ilícito; ii) cuando las familias inscritas individualmente $[. .$.$] incumplan los compromisos adquiridos$ sin que medie caso fortuito o fuerza mayor; y iii) cuando se trate de cultivos de uso ilícito sin un responsable identificable de su establecimiento y sustitución voluntaria. (Defensoría del Pueblo, 2017)

En los dos períodos de Gobierno se han adelantado jornadas de erradicación forzosa por vía manual y aspersión terrestre con glifosato en los municipios priorizados por el PNIs. Desde agosto de 
2017, existe el Puesto de mando táctico y estratégico para la coordinación y seguimiento de la erradicación de cultivos ilícitos ${ }^{13}$, donde el sector defensa y la Dirección han trabajado en la articulación entre el Programa y la erradicación forzada. Sin embargo, en los territorios esta labor no se ha reflejado plenamente, pues casi en la totalidad de municipios PNIS con acuerdos colectivos y vinculaciones individuales se presentaron jornadas de erradicación forzada entre enero de 2017 y diciembre de 201914. Por ejemplo, Tumaco (Nariño) y Tarazá (Antioquia) fueron los que mayores hectáreas erradicadas registraron por vía manual (Observatorio de Drogas de Colombia, 2020), así como son los que actualmente concentran aproximadamente el $20 \%$ de los núcleos familiares vinculados al PNIS.

Dicha descoordinación ha influido en la ocurrencia de movilizaciones, protestas e incluso confrontaciones entre la Fuerza Pública y las comunidades en todo el período de estudio de este documento. Según la Defensoría del Pueblo (2017) durante la vigencia 2017 “[...] en los departamentos de Antioquia, Cauca, Caquetá, Guaviare, Meta, Nariño, Norte de Santander, Putumayo y Vichada [intervinieron] de manera concurrente los programas de erradicación forzosa y sustitución voluntaria de cultivos de uso ilícito". Uno de los hechos emblemáticos acaecidos en este período fue la muerte de siete campesinos en octubre de 2017, en la vereda El Tandil de Tumaco (Nariño), en medio de jornadas de erradicación forzada a cargo de miembros de la Policía Antinarcóticos (Garzón y Suárez, 2018).

Asimismo, solo en los primeros cien días del Gobierno de Iván Duque, las familias cocaleras de municipios de Norte de Santander, Nariño y Caquetá se movilizaron y protestaron en contra del avance de la erradicación forzada por considerarlo una afectación

13 Creado con la Directiva Permanente.$^{\circ} 0032$ del 30 de agosto de 2017 del Ministerio de Defensa Nacional.

14 El único municipio que en el Sistema de Información de Drogas de Colombia (Sidco) no registra cifras de erradicación forzada por vía manual a lo largo de dicho período es Jambaló (Cauca). 
directa a su economía, un contrasentido hacia lo planteado por el PNIS, al igual que una omisión a la voluntad de las familias que sin éxito intentaron vincularse al Programa (Fundación Ideas para la Paz, 2018; Secretaría General de la Organización de Estados Americanos, 2019). Aunque no se presentaron hechos como el de la vereda El Tandil, la descoordinación entre las estrategias sí se mantuvo como un dinamizador de escenarios de protesta social.

\subsection{Plan de atención inmediata}

En ambos períodos presidenciales el PAI ha sido el componente del PNIS que mayores avances ha alcanzado y del que más información disponible existe ${ }^{15}$. La tabla 1 presenta un paralelo entre los resultados alcanzados en cada uno de los elementos del plan para cultivadores, no cultivadores y recolectores.

Tabla 1. Avances en cada elemento del PAl ${ }^{16}$

\begin{tabular}{|lll|}
\hline \multicolumn{1}{|c}{ Categoria } & \multicolumn{1}{c|}{ Periodo Santos } & \multicolumn{1}{c|}{ Período Duque } \\
\hline Municipios priorizados & $\begin{array}{l}52 \text { municipios } \\
\text { (13 departamentos) }\end{array}$ & $\begin{array}{l}56 \text { municipios } \\
\text { (14 departamentos) }\end{array}$ \\
Total familias inscritas & 83161 familias & 99097 familias \\
Hectáreas vinculadas al PNIS & 49094 ha & 60087 ha \\
$\begin{array}{l}\text { Hectáreas vinculadas al PNIS } \\
\text { que fueron erradicadas }\end{array}$ & 26219 ha & 41370 ha \\
\hline
\end{tabular}

15 Debido a los reportes de monitoreo y verificación que bimensualmente elabora unODc y a los informes que desde el año 2020 publica la Dirección de Sustitución de Cultivos llícitos 16 Se toma como referencia el Informe Ejecutivo Consolidado n. ${ }^{\circ} 13$ sobre el monitoreo y verificación del PNIS elaborado por UNODC, el cual tiene fecha de corte el 20 de agosto de 2018. Si bien, trasciende por unas semanas el cierre del Gobierno Santos, este período puede considerarse como un momento de transición entre los dos mandatos presidenciales donde las cifras de ejecución no sufren grandes modificaciones. 
Tabla 1. Avances en cada elemento del pal (continuación)

\begin{tabular}{|c|c|c|}
\hline Categoría & Período Santos & Período Duque \\
\hline \multicolumn{3}{|c|}{ Familias cultivadoras y no cultivadoras } \\
\hline $\begin{array}{l}\text { Número de familias } \\
\text { inscritas }\end{array}$ & $\begin{array}{l}75250 \text { familias } \\
\text { (cultivadoras: } 56465 \text {; } \\
\text { no cultivadoras: } \\
18785 \text { ) }\end{array}$ & $\begin{array}{l}82237 \text { familias } \\
\text { (cultivadoras: } 67237 \text {; } \\
\text { no cultivadoras: } \\
15 \text { 000) }\end{array}$ \\
\hline $\begin{array}{l}\text { Familias con al menos un } \\
\text { desembolso de asistencia } \\
\text { alimentaria }\end{array}$ & 57754 familias & 73332 familias \\
\hline $\begin{array}{l}\text { Familias con el total de } \\
\text { desembolsos de asistencia } \\
\text { alimentaria }\end{array}$ & O familias & 49323 familias \\
\hline $\begin{array}{l}\text { Familias con proyectos de } \\
\text { seguridad alimentaria }\end{array}$ & 6623 familias & 58846 familias \\
\hline $\begin{array}{l}\text { Familias con asistencia } \\
\text { técnica integral }\end{array}$ & 29552 familias & 61183 familias \\
\hline $\begin{array}{l}\text { Familias proyectos producti- } \\
\text { vos formulados }\end{array}$ & 0 familias & 8838 familias \\
\hline $\begin{array}{l}\text { Familias con proyectos } \\
\text { productivos en ejecución }\end{array}$ & 0 familias & 726 familias \\
\hline \multicolumn{3}{|c|}{ Población recolectora } \\
\hline $\begin{array}{l}\text { Número de personas } \\
\text { inscritas }\end{array}$ & 7911 personas & 16860 personas \\
\hline $\begin{array}{l}\text { Recolectores empleados en } \\
\text { obras comunitarias }\end{array}$ & 965 recolectores & 5701 recolectores \\
\hline
\end{tabular}

Fuente: elaboración propia a partir del Informe de Gestión de la Consejería con corte 31 de diciembre de 2019 y de los Informes Ejecutivos Consolidados n. ${ }^{\circ} 13$ y 20 de UNODC.

Si se analiza el Programa desde su aporte a la reducción del área sembrada, la sensación es alentadora. La UNODC (2020) reportó que entre 2017-2019 hubo un progreso del 68,8 \% en la erradicación de las hectáreas acogidas al proceso de sustitución, con un porcentaje de resiembra del 0,4\%. Igualmente, a la luz del Plan nacional de desarrollo vigente (Gobierno de Colombia, 2018), las 
41370 hectáreas erradicadas representan un avance del $80 \%$ en la meta de reducir 50000 hectáreas de cultivos ilícitos vía sustitución hasta el año 2022.

La tabla 1 evidencia un salto cuantitativo en el período Duque frente a los adelantos alcanzados en el período Santos, pues la implementación del PAI entre los últimos meses de 2018 y el 2019 tuvo progresos en cada uno de sus elementos. Por ejemplo, en la ejecución del apoyo de asistencia alimentaria inmediata el $60 \%$ de las familias cultivadoras y no cultivadoras completaron los seis ciclos de pago. Aun así, los avances son diferenciados en los municipios priorizados y los retrasos entre la asignación de los componentes han sido una constante en los dos períodos de Gobierno.

El malestar generado ha movilizado a la población beneficiaria para exigir celeridad en la implementación. Entre febrero y septiembre de 2017, la Defensoría (2017) identificó la ocurrencia de protestas de las comunidades demandando al Gobierno Santos avances en la ejecución del PNIS en municipios como Jambaló (Cauca), Tibú (Norte de Santander), Cáceres y Tarazá (Antioquia) y Cartagena del Chairá, y El Paujil (Caquetá). De manera similar, la Misión de Paz de la Organización de Estados Americanos (MAPP/OEA) (2019; 2020) refirió en sus informes semestrales XXVII y XXVIII que los campesinos cocaleros de los municipios del sur de Córdoba (Montelíbano, Puerto Libertador, San José de Uré y Tierralta), Antioquia (Ituango y Tarazá), Caquetá (San José del Fragua, Puerto Rico, La Montañita) y Putumayo (Puerto Asís, San Miguel y Valle del Guamuez) le exigieron a lo largo de 2019 al Gobierno Duque agilizar la operación de los componentes del PNIS.

A medida que se revisa cada uno de los componentes del PAI para cultivadores y no cultivadores, se denota un estrechamiento entre las cifras de avance. Esto representa una brecha cualitativa entre la finalización de los desembolsos de asistencia inmediata, la entrega de insumos para los proyectos productivos y la formalización de tierras, recursos que apuntan a la estabilización económica de las familias beneficiarias, siendo una de las claves del proceso de transición (Secretaría General de la Organización de Estados Americanos, 2019). El Instituto Kroc (Iniciativa Barómetro, 2020) 
coincide al comentar que, a finales del año 2019, varios compromisos a corto plazo del punto 4 estaban en fase inicial, identificándose los retrasos más importantes en las intervenciones tempranas para el desarrollo de las comunidades PNIS ${ }^{17}$.

La brecha también está entre la atención mínima de la población recolectora y los niveles de avance que ha alcanzado el PAI en los núcleos de cultivadores y no cultivadores. El efecto de dicho desbalance se ha percibido en departamentos como "Arauca, Caquetá y Putumayo, donde la población se ha trasladado hacia zonas con presencia de cultivos ilícitos para retomar las labores de recolección de hoja de coca ante la ausencia de otras fuentes de empleo" (Secretaría General de la Organización de Estados Americanos, 2019). Medidas como el enrolamiento de 754 antiguos recolectores en cursos de formación técnica dictados por el Servicio Nacional de Aprendizaje (SENA) (UNODC, 2020) representan un aporte significativo a su proceso de reconversión laboral y un valor agregado de la implementación del PNIS, pues no están consignadas explícitamente en el Acuerdo de $\mathrm{Paz}^{18}$.

Por último, sobre el adelanto de acciones relacionadas con los elementos de beneficio comunitario del PAI y las obras de interés social, no se encontraron avances en ninguno de los dos períodos estudiados. La única información relevante es que las estrategias se enmarcarán en la implementación de los Programas de Desarrollo con Enfoque Territorial (PDET) en los casos de coincidencia, y en los que no serán responsabilidad de los PISDA (Consejería Presidencial para la Estabilización y la Consolidación, 2019).

\subsubsection{Plan de formalización de la propiedad}

En el período Santos no se adelantaron estrategias de formalización de la propiedad que se enmarcaran en el proceso de im-

17 El Instituto Kroc agrupó temporalmente los compromisos contemplados en el Plan Marco de Implementación del Acuerdo de Paz. Estableció para su seguimiento que las fechas de finalización de las tareas se proyectan a corto plazo (2017-2019), mediano plazo (20202022) y largo plazo (2023-2031).

18 Véanse las páginas 112-113 del Acuerdo de Paz. 
plementación del PNIs. Esto no significa que el Gobierno no haya desarrollado medidas para formalizar predios en las zonas vulnerables a la presencia de cultivos ilícitos, pues desde el año 2016 la Agencia Nacional de Tierras (ANT) ha ejecutado el programa Formalizar para sustituir con el objetivo de regularizar la propiedad rural para mitigar la proliferación de las plantaciones ilícitas. Al respecto, Garzón y Riveros (2018) señalaron que un hecho llamativo en el desarrollo de la estrategia de formalización ha sido:

[...] su baja coincidencia territorial con el programa de sustitución: solo una cuarta parte de los municipios que tienen familias inscritas en el PNIS fueron focalizados por la ANT, mientras que hay municipios en los que se encuentra trabajando la Agencia que no hacen parte del PNIS.

La desarticulación entre las estrategias no permite considerar a los avances del programa Formalizar para sustituir durante la administración Santos como un elemento que aportó al progreso del PNIS.

Por su parte, el Gobierno Duque en la vigencia 2019 adelantó el esquema de coordinación entre dichos programas; proyectó la atención de 1500 familias en los municipios PNIS de San Andrés de Tumaco, Puerto Asís y Tibú a partir del año 2020 (Gobierno de Colombia, 2020). La gestión es el punto de partida para generar acciones conjuntas entre la Dirección y la ANT, aunque para el período de estudio no se presentaron avances materiales en la regularización de la tierra para la población del Programa.

El complemento del proceso de sustitución con las medidas de acceso y formalización de la tierra es vital para que el abandono de los cultivos se sostenga en el tiempo, en especial frente al inicio de los proyectos productivos del PAI y la proximidad al cumplimiento de la meta de erradicación mediante el proceso de sustitución. La tarea no es menor, pues, como lo diagnosticaron la FIP y la UNODC en agosto de 2018, de una muestra de 6350 familias vinculadas al PNIS, el $59 \%$ de los encuestados se consideraban dueños de su predio, pero solo $13 \%$ contaban realmente con escritura pública o sentencia judicial. La diferencia entre la 
respuesta y el estado formal de titularidad se asocia a que las familias consideran el tiempo de ocupación como el factor que los hace acreedores de la propiedad de sus predios, sin importar si tramitaron ante el Estado su legalización. En este sentido, la consideración de estrategias pedagógicas para debilitar la cultura de informalidad — que ha permeado este desafío clave para el desarrollo rural- podría representar un valor agregado para los procesos de formalización y sustitución.

\section{3. Planes integrales de sustitución y desarrollo alternativo}

Durante el período Santos, los informes de gestión del Dapre coinciden en mencionar la ausencia de avances en la construcción de los Pisda. La desarticulación de la Dirección con las agencias del sector agricultura y desarrollo rural — como la ART, ANT y la Agencia de Desarrollo Rural- fue una característica de las acciones en esta línea, lo cual dejó en los territorios la percepción de que el Programa era un asunto aislado a los componentes de la RRI. Por ejemplo, en el 2017 la Oficina en Washington para Asuntos Latinoamericanos (Wola), el Transnational Institute y el Observatorio de Cultivos Declarados Ilícitos identificaron que:

[...] las comunidades no [percibían] coordinación entre el PDET ${ }^{19}$ y el PNIs, hecho que se [reflejaba] más específicamente en la descoordinación entre el Pisda y el PDET. [...] Incluso en algunos sitios donde se [inició] la presencia del programa PDET no se [relacionó] con el tema de la sustitución de cultivos. (Wola, TNI y Ocdi, 2017)

19 Se definen como un instrumento especial de planificación y gestión a 15 años, que apunta a llevar los componentes de la RRI en los 170 municipios más afectados por el conflicto armado, con mayores índices de pobreza, presencia de economías ilícitas y debilidad institucional. Los municipios se agrupan en 16 subregiones según el Decreto 893 de 2017. Cada PDET se instrumentaliza en Planes de acción para la transformación territorial construidos mediante un ejercicio de planeación participativa en el que se identifican y priorizan las necesidades e iniciativas para cada subregión (Agencia de Renovación del Territorio, 2019). 
Por el contrario, a finales de 2018 en el marco de la administración Duque sí se presentaron adelantos en la construcción de los Pisda. En primera instancia, el Ejecutivo:

[...] realizó una revisión de los municipios PDET y los municipios PNIS, encontrando una coincidencia en 48 de los 56 municipios objeto de intervención y una identificación de 728 iniciativas PDET. Con ello, se inicióla construcción de los documentos Pisda, los cuales fueron finalizados a diciembre de 2019. (Consejería Presidencial para la Estabilización y la Consolidación, 2019)

En segunda instancia, determinó la ruta para los ocho municipios PNIS que no coincidían con los PDET, ejercicio que se llevó cabo en la vigencia 2020. En sí, queda la tarea de desarrollar las más de 700 iniciativas que se han proyectado para la transformación de los 56 municipios cocaleros y de las condiciones de vida de sus habitantes.

\subsection{Tratamiento penal diferencial}

Los avances en este componente son nulos, pues hasta diciembre de 2019 no existía una ley de tratamiento penal diferencial en firme. Entre enero de 2017 y agosto de 2018, el Gobierno Santos tramitó sin éxito dos proyectos de ley sobre el tema en cuestión: el primero el 24 de octubre de 2017 y el segundo el 21 de marzo de 2018 (Sistema integrado de información para el posconflicto, 2020). En las dos oportunidades, la controversia estuvo en la definición de lo que legalmente se consideraría como pequeño cultivador, teniendo como criterio el número de hectáreas cultivadas con plantaciones ilícitas.

Por su parte, entre agosto de 2018 y diciembre de 2019 el Gobierno Duque no presentó ningún proyecto de ley sobre este tema. La ausencia de avances en este asunto debilita la estructura normativa del PNIS al no existir definiciones legales mínimas que brinden seguridad jurídica a la población beneficiaria de la sustitución. 


\subsection{Condiciones de Seguridad para la sustitución DE CULTIVOS ILÍCITOS}

En el Gobierno Santos no se implementaron estrategias específicas de protección para la población PNIs. En contraste, en el año 2019 la administración Duque construyó, en conjunto con los representantes de las comunidades beneficiarias, el Plan de articulación de acciones de seguridad para la población objeto del PNIs liderado por la Consejería y coordinado con las entidades con funciones en materia de protección de población especial, seguridad ciudadana e investigación judicial ${ }^{20}$.

La existencia de este plan es una respuesta a los riesgos que los líderes, beneficiarios y el personal de las entidades involucradas en el desarrollo del PNIS han afrontado durante el período estudiado. En el año 2018, la MAPP/ OEA reportó que el hecho de que el PNIS ofrezca proyectos que sustituyan la dependencia de las familias de una economía ilegal, lo que implica la llegada institucional. Además, se configura un escenario de riesgo para los lideres promotores del Programa y la población beneficiaria, debido a la presencia de actores armados ilegales que encuentran en los cultivos ilícitos una fuente de financiación para su accionar (Secretaría General de la Organización de Estados Americanos, 2018). El dossier de grupos que operan en Colombia es amplio, entre los principales se encuentran el Ejército de Liberación Nacional (ELN), el Clan del Golfo, el Ejército Popular de Liberación (EPL) y las distintas facciones de las disidencias de las FARC-EP (Gobierno de Colombia, 2019) que han asumido posturas y acciones diferentes con respecto a la entrada del Programa a los territorios.

Tanto en el mandato Santos como en el de Duque, la ejecución de los componentes del PNIS se ha visto afectada por el desarrollo de estrategias generales de control territorial de los grupos armados

20 La Unidad Policial para la Edificación de la Paz (Unipep) y el Cuerpo Élite de la Policía Nacional, el Comando Conjunto Estratégico de Transición (cCOET) de las Fuerza Militares, la Unidad Nacional de Protección, la Unidad de Especial de Investigación de la Fiscalía General de la Nación. 
ilegales o su oposición a la vinculación de las comunidades al esquema de sustitución en los municipios focalizados de Antioquia, Córdoba, Caquetá, Cauca, Guaviare, Meta, Nariño y Putumayo. Lo anterior, incluso en los núcleos veredales que no superaron la fase de acuerdos colectivos. Sin entrar en precisiones cuantitativas, las afectaciones contra personas que promueven o participan de la sustitución son diversas: homicidios, desplazamientos forzados, amenazas, extorsiones a los recursos asignados, restricciones a la participación en las instancias del PNIS, entre otras (Secretaría General de la Organización de Estados Americanos, 2019).

Igualmente, las instituciones involucradas en la implementación han visto limitadas sus funciones y el trabajo de su personal por el complejo contexto de seguridad. El caso del secuestro del funcionario de UNODC en Miraflores (Guaviare) en 2017-liberado el mismo año- (Noticias ONU, 2017) y los obstáculos para la realización de las visitas de monitoreo y verificación a finales de 2019 en veredas de los municipios de San José del Guaviare, Calamar, El Retorno, Miraflores (Guaviare), Puerto Concordia, Mapiripán y Puerto Rico (Meta) son ejemplos de ello (UNODC, 2020).

Desde el 2018, una de las medidas adoptadas para contrastar lo anterior ha sido el empleo de la fuerza pública en el monitoreo de la erradicación de algunos territorios donde la UNODC no pudo desplazarse; al igual que la puesta en marcha de la erradicación asistida para apoyar a las familias en el cumplimiento de sus compromisos (Garzón y Gélvez, 2018 B). Ambas estrategias han sido mantenidas por el Gobierno Duque por su contribución al cumplimiento de las metas de reducción del área sembrada vía sustitución y a la mitigación de los riesgos a la seguridad de las familias PNIS en territorios donde los grupos armados ilegales se oponen al levantamiento de los cultivos ilícitos.

Durante el período de estudio, la inestabilidad de las condiciones de seguridad se gestó, según la MAPP/OEA (Secretaría General de la Organización de Estados Americanos, 2018), en un primer momento, en torno a la activación de disputas armadas entre los grupos armados ilegales por los territorios dejados por las FARC-EP; en un segundo momento, por las dinámicas de reacomodo, consolidación 
y expansión territorial. Para la FIP (Bernal, Garzón y Gélvez, 2019), la violencia persistente también responde a los rezagos en el proceso de reincorporación de los excombatientes, a la escasa presencia estatal sostenida en el tiempo, a las dificultades en la implementación de las garantías de seguridad contempladas en el Acuerdo de Paz y a la existencia de múltiples economías ilegales, así como a la concentración de cultivos en zonas no intervenidas por el PNIS y a la existencia de enclaves productivos que colindan con las veredas en proceso de sustitución. Ante este contexto, la protección de la población es clave para garantizar su permanencia en la ruta del Programa.

\subsection{Sustitución en Parques Nacionales Naturales}

Entre enero de 2017 y agosto de 2018, el desarrollo de estrategias para la restauración y conservación de los Parques Nacionales Naturales afectados por la presencia de cultivos ilícito en el marco del PNIs no tuvo avances representativos. Según el Sistema integrado de información para el posconflicto (2020), lo único adelantado en este componente fue la vinculación de familias con cultivos de hoja de coca al interior del Santuario de Flora y Plantas Medicinales Orito Ingi Ande - ubicado en el departamento de Putumayo-y la gestión de acciones de restauración.

En contraste, la FIP señaló en su más reciente informe sobre cultivos ilícitos y Parques Nacionales Naturales que, solo hasta finales del 2018, bajo el mandato de Iván Duque, la Dirección de Sustitución y la Unidad Administrativa de Parques Nacionales Naturales formularon un protocolo de trabajo para la implementación del PNIS en las siguientes áreas protegidas: Alto Fragua Indi Wasi (Caquetá), Farallones de Cali (Valle del Cauca), Santuario de Flora y Plantas Medicinales Orito Ingi Ande y La Paya (ambos en Putumayo), Munchique (Cauca), Paramillo (entre Córdoba y Antioquia), Sierra de La Macarena y Tinigua (ambos en Meta) (Bernal, Garzón y Riveros, 2020). Bajo esta focalización, al Gobierno le queda un gran trabajo para lograr en la práctica la articulación entre la política de drogas y la política ambiental sobre la base de lo adelantado en el proceso de sustitución. 


\section{Conclusiones}

Retomando la pregunta de investigación central: ¿en qué sentido lo implementado del PNIs ha contribuido o no al proceso de construcción de paz en Colombia, en especial con respecto al fomento del desarrollo rural? Lo expuesto permite confirmar que el Programa no se ha consolidado como estrategia de transición que aporte a la transformación del campo colombiano, debido a que los componentes del vehículo para dicho fin se encuentran aún en fase embrionaria. Para que el alcance del pNIS no se limite al de una estrategia de contención, tanto el Gobierno actual, como los siguientes, requerirán de esfuerzos para mejorar su eficiencia y consolidar su articulación con la RRI.

Ante el interrogante de cuál ha sido entonces el desempeño del PNIS en sus dos años de ejecución, se concluye que el Programa ha sido suficiente en la reducción de la cantidad de hectáreas de hoja de coca cultivadas en el país, pero insuficiente en la provisión de respuestas para el mejoramiento sostenido de la calidad de vida de la población cocalera y el desarrollo rural. Si bien es cierto que al Programa le queda por delante atender asuntos de mayor envergadura - como el desarrollo de los proyectos productivos de ciclo largo, afianzar los procesos de formalización de la propiedad rural, reforzar la provisión de bienes y servicios comunitarios, entre otros-, los componentes implementados en el período de estudio han forjado las bases de las siguientes fases del PNIS, lo que refleja los elementos por mantener y aquellos que requieren ajustes.

Aquí se enlaza la última pregunta planteada por este documento: ¿cuáles han sido los avances y dificultades del PNIs? Durante el período de estudio, los mayores avances se concentraron en la ejecución de los componentes iniciales del PAI familiar para la población cultivadora y no cultivadora - asistencia inmediata y seguridad alimentaria-; en la erradicación casi total de las hectáreas de hoja de coca sometidas al proceso de sustitución; en el fortalecimiento estructural de la ruta de intervención del Programa; y en el formulación de la mayoría de instrumentos de planeación 
que engranan al PNIS con los PDET, es decir, los Pisda. Aun así, las dificultades afrontadas en los dos períodos de Gobierno impactaron en la credibilidad y confianza de los beneficiarios hacia el proceso de sustitución y hacia el Estado en general. La configuración de las actividades del Programa como un escenario de riesgo a la seguridad física de su población objeto y los desfases en la ejecución de los componentes del PAI familiar fueron los principales factores que llevaron a ello.

El conjunto de familias beneficiarias del PNIS está probablemente entrando en lo que la UNODC (Simci, 2020) ha descrito como la etapa de tensión dentro de la dinámica de abandono de los cultivos ilícitos. En este momento, la erradicación ha alcanzado un porcentaje de avance cercano al $100 \%$, pero perturbaciones como las mencionadas anteriormente configuran la amenaza de resiembra. Quizás esta será la fase que deberá sortear el Gobierno nacional durante el resto de su mandato, para la cual requerirá no solo de estrategias para agilizar la ejecución del PNIS, sino también de canales de diálogo incidentes para reforzar la confianza y legitimidad del Estado en los territorios cocaleros.

Para futuras investigaciones queda la tarea de ahondar en los desafíos que afrontará el Estado colombiano para llevar a buen puerto el proceso de sustitución. El presente estudio permite prever que las acciones para la inclusión social y productiva de los territorios implicarán aún más capacidad del aparato estatal que lo ejecutado hasta ahora, en especial a la hora de adelantar las estrategias para la provisión de bienes públicos y la formalización de la propiedad rural. Sin estos elementos, es probable que el abandono de los cultivos no sea una situación sostenida en el tiempo, se abra paso a la resiembra y, con ello, a la persistencia de los desafíos para la seguridad y el desarrollo que se configuran en torno a la producción de cultivos de hoja de coca para fines ilícitos.

\section{REFERENCIAS}

Agencia de Renovación del Territorio. (2019). ABC Ruta de construcción de los Programas de Desarrollo con Enfoque Territorial-PDET. bit.ly/3lrgWPq 
Álvarez Vanegas, E. y Garzón, J. C. (2017). ¿En qué va la sustitución de cultivos ilícitos? Principales avances, desafíos y propuestas para hacerles frente. Fundación Ideas para la Paz, Bogotá.

Álvarez Vanegas, E. y Garzón, J. C. (29 de octubre de 2017). ¿En qué va la sustitución de cultivos ilícitos? Principales avances, desafíos y propuestas para hacerle frente. Informe trimestral 2. https: / / bit.ly/38Fkf04

Archivo de la Presidencia 2015-2018. (27 de enero de 2017). Listo plan para sustitución voluntaria de cultivos ilícitos. https: / / bit.ly / 3vrHlB9

Bernal, J. L. y Garzón J. C. (4 de julio de 2017). ¿En qué va la sustitución de cultivos ilícitos? Principales avances, desafíos y propuestas para hacerle frente. Informe trimestral 1. https: / bit.ly/2OEnqhE

Bernal, J. L., Garzón, J. C. y Gélvez, J. D. (2019, abril). ¿En qué va la sustitución de cultivos ilícitos? Desafíos, dilemas actuales y la urgencia de un consenso. Informe trimestral 6. https: / / bit.ly/38LQIC6

Bernal, J. L., Garzón, J. C. y Riveros, C. (2020). Cultivos ilícitos y áreas protegidas del Sistema de Parques Nacionales Naturales. Bogotá. https:/ / bit.ly / 3eHE9eL

Consejería Presidencial para la Estabilización y la Consolidación. (2019). Informe de Gestión PNIS a 31 de diciembre de 2019. https: / bit.ly / 3limfjU

Consejo Nacional de Política Económica y Social. (2018). Conpes 3932 Lineamientos para la articulación del Plan Marco de Implementación del Acuerdo Final con los instrumentos de planeación, programación y seguimiento a políticas públicas del orden nacional y territorial. https: / / bit.ly / 3eIuODE

Defensoría del Pueblo. (2017). Análisis de la fase inicial de diseño e implementación del Programa Nacional Integral de Sustitución de Cultivos de Uso Ilícito-PNIS. Bogotá. https: / / bit.ly/3cCC94D

Departamento Administrativo de la Presidencia de la República. (2017). Informe Técnico de Rendición de Cuentas 2016-2017. https: / / bit.ly/2Q88jxB

Departamento Administrativo de la Presidencia de la República. (2018). Informe Técnico de Rendición de Cuentas 2017-2018. https: / / bit.ly /30NM4PE

Departamento Administrativo Nacional de Estadística. (2019). Pobreza Multidimensional por Departamentos 2018. https: / / bit.ly / 2OXJmV3

El Tiempo. (2019, 3 de diciembre). El pueblo azotado por las Farc que se convirtió en el municipio 1.103. http:/ / bit.ly/30MS5Mg

Fundación Ideas para la Paz y unOdC. (2018). ¿Quiénes son las familias que viven en las zonas con cultivos de coca? Bogotá. https: / / bit.ly /3vAMYx9

Fundación Ideas para la Paz. (2014). Solución al Problema de las Drogas Ilícitas. Boletín de Paz n. ${ }^{\circ} 34$ - Negociaciones de Paz. https: / / bit.ly / 3ljdEO0

Fundación Ideas para la Paz. (2018). Los primeros 100 días del presidente Iván Duque. https: / / bit.ly/3cDkR7y

Garzón, J. C. y Gélvez, J. D. (2018 A). ¿En qué va la sustitución de cultivos ilícitos? La implementación, los rezagos y las tareas pendientes. Informe trimestral 4. https: / / bit.ly/38ZQ04n 
Garzón, J. C. y Gélvez, J. D. (2018 B). ¿En qué va la sustitución de cultivos ilícitos? Desafíos y recomendaciones para el nuevo gobierno. Informe trimestral 5. https: / / bit.ly/38ZQjMz

Garzón, J. C. y Riveros, C. (2018). Acceso a la tierra y sustitución de cultivos ilícitos. Desafios y oportunidades para el desarrollo rural. Fundación Ideas para la Paz. https: / / bit.ly/3eLCKnp

Garzón, J. C. y Suárez, M. (2018, febrero). ¿En qué va la sustitución de cultivos ilícitos? Balance del 2017 y lo viene en 2018. Informe treimestral 3. https:/ / bit. ly/3llpQxW

Gobierno de Colombia. (2018). Bases del Plan Nacional de Desarrollo 2018-2022. pp. 1224.

Gobierno de Colombia. (2019, enero). Política de Defensa y Seguridad. pp. 22-29. https: / / bit.ly/2OzWDDh

Gobierno de Colombia. (2020). Avanza la sustitución con legalidad. Diagnóstico, planeación y avances PNIS a 31 de junio de 2020. https: / / bit.ly/3eIPICq

Gobierno de Colombia. (2020, 22 de agosto). Casos positivos de Covid-19 en Colombia. http: / / bit.ly/30NZYkw

Iniciativa Barómetro. (2020). Tres años después de la firma del Acuerdo Final de Colombia: hacia la transformación territorial. Informe 4, Universidad de Notre Dame, Instituto Kroc de Estudios Internacionales de Paz.

Mejía, D. y Rico, D. (2010, 19 de julio). La microeconomía de la producción y tráfico de cocaína en Colombia. Documentos CEDE.

Mesa de Conversaciones de Paz. (2017). Acuerdo Final para la Terminación del Conflicto y la Construcción de una Paz Estable y Duradera.

Noticias ONU. (2017, 4 de mayo). Funcionario de la ONU secuestrado en Colombia será liberado en breve. http: / / bit.ly/3vD4GQy

Observatorio de Drogas de Colombia. (2020, junio). Sistema de Información de Drogas de Colombia. http:/ / bit.ly/3bRMjiE

Oficina del Alto Comisionado para la Paz. (2020, 30 de junio). Base de datos de Victimas por MAP/MUSE. http: / / bit.ly/3vpVkr3

Pardo Rueda, R. (2020). La Guerra Sin Fin. Bogotá: Aguilar.

Presidencia de la República. (2017, 29 de mayo). Decreto Ley Número 896 de 2017. https: / / bit.ly/3eI1flt

Presidencia de la República. (2019). Decreto 179 del 08 de febrero de 2019.

Secretaría General de la Organización de Estados Americanos. (2018). Vigésimo Quinto Informe del Secretario General al Consejo Permanente sobre la Misión de Apoyo al Proceso de Paz en Colombia de la Organización de los Estados Americanos (MAPP/OEA).

Secretaría General de la Organización de Estados Americanos. (2019). Vigésimo Séptimo informe del secretario general al Consejo Permanente sobre la Misión de Apoyo al Proceso de Paz en Colombia de la Organización de Estados Americanos (MAPP/OEA). 
Secretaría General de la Organización de Estados Americanos. (2020). Vigésimo Octavo Informe del Secretario General al Consejo Permanente sobre la Misión de Apoyo al Proceso de Paz en Colombia de la Organización de Estados Americanos (MAPP/OEA).

SIMCI. (2019). Monitoreo de Territorios Afectados por Cultivos Ilícitos 2018.

SIMCI. (2020). Monitoreo de Territorios Afectados por Cultivos Ilícitos 2019.

Sistema integrado de información para el posconflicto. (2020, 30 de junio). Avance de indicadores por estrategia: Punto 4 Solución al problema de las drogas ilícitas. http: / / bit.ly/30N5Hak

Soto, L. (2020, 17 de julio). En medio de la incredulidad, Duque apuesta por nueva estrategia de sustitución de coca. http: / / bit.ly / 3vtsXIE

UNODC. (2018). Informe Ejecutivo Consolidado n. ${ }^{\circ} 13$ Programa Nacional Integral de Sustitución de Cultivos Ilícitos-PNIS.

UNODC. (2020). Informe Ejecutivo Consolidado n. ${ }^{\circ} 20$ Programa Nacional Integral de Sustitución de Cultivos Ilícitos-PNIS. Monitoreo a la implementación del Plan de Atención Inmediata - componente familiar.

WOLA, TNI y OCDI. (2017, 11 de noviembre). Diálogo informal sobre "Avances y desafíos en la implementación del Punto 4 del Acuerdo de Paz". Bogotá. 